\title{
The Polymath Questionnaire: A Correlational Study between Polymath Belief, Self-Efficacy, and Personality
}

\author{
Shannon J. Fernandes \\ St. Xavier's College, Mumbai ${ }^{1}$ \\ fernandesshannon.sf11@gmail.com
}

\begin{abstract}
This study attempts to explore the idea of a 'polymath' and treats education and learning as an end in itself, rather than a means. The paper focuses on the relationship between polymath beliefs, a sub-type of self-efficacy, i.e. the extent to which an individual finds themselves to be competent to carry out the different tasks in several areas. The major distinction between self-efficacy as an umbrella term and the Polymath Belief is that the latter focuses on one's perceptions in more than one areas, while the former isn't particularly dependent on the number of fields/areas pursued. The Polymath Questionnaire was constructed to assess polymath belief, which was correlated with Openness and Self-Efficacy. The study found significant positive correlations. The paper concludes with a discussion on the merits and limitations of the study, and scope for further research.
\end{abstract}

\footnotetext{
${ }^{1}$ This paper was written for and presented at the Psychology Seminar (2021) at St. Xavier's College,
} Mumbai. 


\section{Introduction}

"Having a large measure of one good quality increases the probability that one will have more than the average of any other good quality"

- Thorndike (1911)

A Polymath, known as the 'Renaissance Man' before, is an individual with varied interests. A Polymath is seen as a genius in some cultures but more importantly as important figures, innovators or wise individuals who do not limit themselves to a particular field. History has provided us with such figures from the ancient Greece like Aristotle who delved into various branches of study like philosophy, metaphysics, anatomy, physics, among others (Barnes, 1995), to the renowned Italian figure, Leonardo Da Vinci, most famously known to have painted the Mona Lisa among other paintings but also contributed to anatomy and engineering, to someone a bit more close to home like Rabrindranath Tagore who wrote poems, plays, and even composed pieces while dealing with social issues. However, the realm of psychology has given this area relatively less attention. It is the view of the author that these polymaths are vital in understanding intelligence and all that it contains, and thus require more attention.

Modern cinema and pop-culture seem to understand this importance as well, although presented subtly, a notion that lurks in the background and not made verbal. The characters we see, often those who carry the 'genius' archetype, are seen as having more than one interest. Rick Sanchez, for example, from the popular show Rick \& Morty, is a scientist who is considered to be the smartest human in the universe and he delves in most areas of science like biology to chemistry to physics and he even creates his own artificial general intelligence. This pattern goes on in various popular shows or movies, running through famous characters like the Batman in The Dark Knight trilogy or Hermoine Granger from the Harry Potter series or even Amy Santiago from the beloved show Brooklyn Nine-Nine. These characters either form a separate archetype or rather merge with the 'genius' archetype. The distinction is hardly relevant in the realm of psychology as both eventually fall under intelligence. However, these patterns from modern cinema wouldn't suffice to make a logical hypothesis. Thus we would need an a priori syllogism that fits with this pattern and illuminates a broader range of intelligence. Intelligence has several definitions but the one component that seems to repeat itself is the notion of 'problem 
solving'. Problem solving is a key component of intelligence and for good reason as intelligence is seen as an evolutionary advantage to ensure survival (Gabora, L., \& Russon, A., 2011). Survival requires you to solve problems inherently. It is cold and therefore you need to think of a way to produce fire at demand. The animals you hunt have claws and teeth so you create spears that are long to keep a safe distance while even attacking. All of these require some kind of problem solving. We often see geniuses or the 'genius' archetype to be precise with this lens, those who dedicate their lives to a particular field and solve a major issue.

However, this lens is applicable to polymaths as well, as problems rarely present themselves as a single entity but rather as a string of problems, each intertwined with other subjects or fields of study. Thus, the polymath engages with different fields to solve problems that are such and these are often higher order problems as they require expertise over various fields since they jump from one field to another quickly. The subjects were created to study the world and not the other way round, thus the universe has no reason not to present itself in the way it does, one that includes jumping from one field to another. The human brain for example is almost impossible to study through only one field and thus lately more and more fields are joining to study the brain ranging from psychology to neuroscience to even physics and quantum theory (Bohm, D., 2002), providing a fascinating notion called the 'quantum mind'. Thus the polymath is a vital part under the realm of intelligence as it shows the potential we are born with. None of us were born to become doctors, lawyers, or psychologists, we have the potential to be any of these things when we are born and here polymaths prove this point, they show us the extent to which the human brain can extend and the heights at which intelligence can reach when this potential is tapped more than once.

Thorndike (1911) had first mentioned that an individual who is good at a particular field or area of interest is more likely to be good at other things, as these individuals learn better. $\mathrm{He}$ (1911) even went on to emphasize the importance of artistic abilities in the scientific fields and scientific thinking. A classical study in the field, however, comes from Root-Bernstein, et al., (2008) in a paper titled "Arts Foster Scientific Success". In their study they compared the avocations and interests of various esteemed researchers, measured through the prestige of the award received, other than their main focus of interest. They found that Nobel Laureates were $50 \%$ more likely than Royal Society members or the National Academy of Sciences members to have different avocations or interests. 
They found significant differences in the permutations using a chi-square analysis. Further analysis showed that Nobel Laureates were 8 times more likely than an average scientist to be a photographer, and 2 times more likely to be a composer, musician or conductor, 7.5 times more likely to engage in craftwork like a mechanic or woodwork or even electronics, 7 times more likely to be a sculptor, a visual artist, or a printmaker, and 12 times more likely to engage in writing various things like plays, poems, novels among others, and finally 22 times more likely to be a performer like a dancer or a magician or even an actor.

They found these differences to be significant at $p>0.0001$ in most cases. Another study (White, 1931) found that individuals who were categorized as intelligent or gifted were more likely to have different interests, those differing from their main interests.

It is thus hypothesized by the author that higher scores on the polymath belief, i.e. their belief in themselves to engage in different activities or fields, usually those differing from one's main interest, would be be positively correlated with multiple interests variable, the manifestation of the belief in general, and the 'virtual' variable, the manifestation of the belief in the virtual world. The Polymath Belief is a particular type of self-efficacy. The supplementary hypothesis aims to explore correlations between a) the Polymath Questionnaire and Personality, and b) the Polymath Questionnaire and Self Efficacy.

\section{A Critique of Capitalist Education}

The polymath belief is a critique of the capitalist education model. The Capitalist Education model is dependent on generating maximum profit and thus it is in this model's benefit if an individual does not promote polymath belief, for reasons we will explore in a bit. The Capitalist education model has a simple 'learn to earn' motto and students are encouraged to study as hard as possible so they can grow to become a part of the workforce and make more money, i.e. increase profit. This is often hidden under the guise of elitist success as it could describe a particular profession (law, medicine, engineering) as being the goal to strive for to earn enough money. It is beneficial for the capitalist education model to discourage interest in various fields as in pursuing only one field an individual would spend less time learning and more time working, thus again maximizing profit. The focus, in this model, is on climbing the ranks (promotions, earning more, etc.) than on solving problems. The Polymath works and learns for the sake of solving problems and above all for the sake of learning and working, not to earn 
more. The polymath understands the importance of money but perceives the world differently, as transcending monetary value. A Polymath Education Model would ensure scientific progress as individuals would pursue various fields, allowing them to collaborate with such individuals and solve problems faster and efficiently, keeping in mind the various perspectives that exist.

\section{Questionnaire Construction}

The questionnaire consists of three dimensions. The first dimension pertains to the polymath belief, the notion mentioned above as being a particular type of self-efficacy. The Polymath Belief dimension attempts to measure the individual's thought process and beliefs, the extent to which an individual finds themselves to be competent to carry out the different tasks in several fields. The major distinction between self-efficacy as an umbrella term and the Polymath Belief is that the latter focuses on one's perceptions in more than one areas, while the former isn't particularly dependent on the number of fields/areas pursued. This is a phenomenological dimension as it depends on the individual's perception of themselves, especially their abilities. If the individual perceives himself/herself as being competent enough to complete the given tasks, they would score higher on this dimension, it would reflect their true beliefs or perceptions about themselves. The individual's beliefs aren't limited to their own abilities, however, but rather even extended to the outside world and the extent to which and the manner in which it presents itself to the individual. If the individual perceives the external world as being restrictive and divided into categories, the individual would score low on this dimension. A polymath rarely holds this view but rather holds a much more flexible view, one that attempts to incorporate as many factors as possible to solve a particular problem. It is for this reason that a personality inventory was correlated with this questionnaire as the author believes there would be a significant correlation between the openness dimension and this questionnaire, specifically between openness and the 'belief' dimension. Items like "I believe that one can become a genius and isn't determined by their genes or IQ" under this dimension indicate one's flexible view of the world and oneself, as opposed to a restrictive one (in this case would be akin to biological determinism). The second dimension attempts to measure an actual manifestation of the belief dimension. You may believe that you are competent in various fields but to what extent are you actively attempting to engage in these fields or learn more about those fields. Items like "I often 
find myself fascinated by a range of fields or topics that aren't explicitly relevant to my current chosen field" try to probe for real world manifestations of their perceptions or beliefs about themselves. This fascination for different fields is beyond 'belief' but instead related to one's actual attraction to these different fields and thus their attempts to engage with them. The last dimension is similar to the 'interest' dimension but it focuses on the virtual world or virtual manifestation of these beliefs (see discussion for note on further study). The virtual dimension attempts to measure these manifestations in the virtual world and how the individual engages with various tools and resources available to create or learn. Thus items like "I spend a lot of time on sites like Youtube or listen to podcasts, learning about topics that aren't related to the one I have currently chosen" point towards the manifestation of the belief in the virtual realm.

\section{Methodology}

The study administered the Polymath Questionnaire (PQ) to 232 participants using an online software, on which the items were coded (see Appendix), called Psytoolkit (Stoet, G., 2010, 2017). The items on the scale had a 'true' or 'false' pattern that the participants had to respond to after carefully reading the instructions. The form was coded such that the participants could not proceed with the questionnaire unless they had filled every item. Furthermore, it was coded such to ensure the items were presented in a random order to each participant. The form did not note any other information except for the age and gender, it did not note down any personal information to ensure confidentiality. Since it was 'self-administered', the form was coded such that the participants were able to see their score at the end as the form calculated their results. The form held a note before the participant entered that mentioned that filling the form would mean that the participant consented to participate in the study and consented for their data to be used for further research. The reliability of the test was determined using test-retest reliability. The Polymath Questionnaire was presented along with the Ten Item Personality Inventory, which has a reasonable reliability $(r=.72)$ and validity, to determine the correlation between PQ and the openness dimension (S. D. Gosling, P. J. Rentfrow, and W. B. Swann Jr., 2003). The Ten Item Personality Inventory was used to ensure that the participants didn't quit filling out the form due to its length. The form had a self-efficacy scale as well, specifically the Generalized Self-Efficacy scale (Schwarzer, R., \& Jerusalem, M., 1995) which too had a 
reasonable reliability of .80 . The self-efficacy scale was added to find a correlation between self-efficacy scores and the scores on the Polymath Questionnaire, especially the 'belief' dimension as it is considered by the author as a sub-type of self-efficacy.

\section{Results}

The Polymath Questionnaire was correlated with the three dimensions $(n=232)$ and there was a significant positive correlation $(r=.779, p>0.00001)$ between the total score $(M=$ $9.96, S D=1.33)$ and the Belief variable $(M=3.08, S D=0.87)$. There was a significant positive correlation $(r=.613, p>0.00001)$ between the total score and the Interest variable $(M=3.35, S D$ $=.59)$. Lastly there was a significant positive correlation $(r=.552, p>0.00001)$ between the total score and the Virtual variable $(M=3.53, S D=.56)$. Among the three dimensions, there was a significant positive correlation between Belief dimension and Interest Dimension $(r=.193, p=$ .003). There was no significant correlation between the Belief dimension and the Virtual dimension, similarly, there was no significant correlation between the Interests dimension and the Virtual dimension.

Table 1.1 - Correlations Between the Three Dimensions and the Total Score $(n=232)$

\begin{tabular}{lllll}
\hline Dimension & Total Score & Belief & Interests & Virtual \\
\hline Total Score & 1.00 & $.779 *$ & $.613^{*}$ & $.552^{*}$ \\
Belief & $.779^{*}$ & 1.00 & $.193^{*}$ & .095 \\
Interests & $.613^{*}$ & $.193^{*}$ & 1.00 & .095 \\
Virtual & $.552^{*}$ & .095 & .095 & 1.00 \\
\hline
\end{tabular}

With respect to the Personality inventory used, there was a significant positive correlation between openness $(M=5.72, S D=.81)$ and the Polymath Questionnaire $(r=.383, p>0.00001)$. Thus, it would appear that the supplementary hypothesis is supported. Furthermore, there was a 
significant positive correlation between openness and the 'belief' dimension $(r=374, p>$ $0.00001)$. There was a significant positive correlation $(r=.160, p=.014)$ between the Polymath Questionnaire and Emotional Stability $(M=4.26, S D=1.23)$. There was no significant correlation between the Polymath Questionnaire and the rest of the big five dimensions (see the Appendix for the correlational table). With respect to the correlation between the Generalized Self-Efficacy Scale $(M=31.56, S D=5.11)$ and the Polymath Questionnaire, there was a significant positive correlation $(r=252, p=.0001)$.

The reliability of the questionnaire could not be determined using methods of internal consistency as the dimensions, and thereby the items, were heterogeneous. Thus a test-retest method of reliability had to be conducted with a smaller sample size $(n=12)$. The test-retest method found that the scale had a reliability of .84 .

\section{Discussion}

This study aimed at creating a tool to assess polymath belief, i.e. the extent to which an individual believes they are capable of engaging in different fields. The results found significant results between the Polymath Questionnaire and the dimensions. It also found significant results between the Polymath Questionnaire and the Generalized Self-Efficacy Scale (Schwarzer, \& Jerusalem), and the Ten Item Personality Inventory (Gosling, Rentfrow, Swann, 2003). However, the scale has limitations and this study should be seen as a preliminary study in this area. This study would, in the author's opinion, have a moderate internal validity as the items were designed carefully to assess the beliefs of an individual and the results were significant, however, the form administration could not be controlled as it was provided as a link over the internet which would make it difficult to control any confounding variables. The external validity would be low as the sample size is neither adequate nor representative of the population. The experimental realism would range between low and moderate as the task of filling a survey usually requires less active concentration or attention. Lastly, the mundane realism of the study would be moderate as individuals fill forms or surveys often, especially among students, which appears to be the major age group.

The limitations of the study, apart from the confounding variables mentioned above, are

firstly the high mean of the scale $(M=9.96, S D=1.33)$ which could be seen as a 'fact' that 
everyone has some extent of polymath belief or thoughts or more simply the items were skewed in that direction, to ensure the desired results. Thus, further research would be needed to control for this and to select items that are carefully constructed to avoid this. A few items were reversed to control for the acquiescence bias, but it would appear the control wasn't sufficient. Furthermore, this would also indicate that the items weren't able to discriminate between high scorers and low scorers and thus the revised scale would require to take this into account by subjecting the items to a thorough item analysis to identify the poor or non-discriminating items. Lastly, the sample size of the test-retest reliability was too small to be adequate or representative. The purpose of this study was to make the notion of polymath belief known so that individuals, especially educators, could incorporate the idea to tailor holistic education, unlike the capitalist education model we critiqued. Further research would be required in this area to make generalizable claims and to measure the polymath belief in the context of different areas.

A psycho-social critique of this study would revolve around the idea of 'privilege'. The study had a sample size that could be considered to be relatively privileged. Individuals that come from well-to-do families are more likely to be introduced to extra-curricular activities and have the purchasing power to enroll in these activities. Furthermore, these individuals have the time available as well to engage with these activities unlike an individual who has to earn to meet his/her daily needs. Thus, further research would be required keeping in mind the varying privilege that exists and the inability of people with low financial resources to engage in these activities and that would probably even influence their polymath belief and self-efficacy. 


\section{References}

Barnes, J. (1995). The Cambridge companion to Aristotle.

Bohm, D. (2002). Wholeness and the implicate order. London: Routledge.

Gabora, L., \& Russon, A. (2011). The evolution of human intelligence. In R. Sternberg \& S. Kaufman (Eds.), The Cambridge handbook of intelligence (pp. 328-350). doi:10.1017/CBO9780511977244.018

Root-Bernstein, R., Allen, L., Beach, L., Bhadula, R., Fast, J., Hosey, C., Kremkow, B., Lapp, J., Lonc, K., Pawelec, K., Podufaly, A., Russ, C., Tennant, L., Vrtis, E., \& Weinlander, S. (2008). Arts foster scientific success: Avocations of Nobel, National Academy, Royal Society, and Sigma Xi members. Journal of Psychology of Science and Technology, 1(2), 51-63. https://doi.org/10.1891/1939-7054.1.2.51

S. D. Gosling, P. J. Rentfrow, and W. B. Swann Jr. (2003). A very brief measure of the Big-Five personality domains. Journal of Research in Personality, 37, 504-528.

Schwarzer, R., \& Jerusalem, M. (1995). Generalized Self-Efficacy scale. In J. Weinman, S. Wright, \& M. Johnston, Measures in health psychology: A user's portfolio. Causal and control beliefs (pp. 35-37). Windsor, UK: NFER-NELSON.

Stoet, G. (2010). PsyToolkit - A software package for programming psychological experiments using Linux. Behavior Research Methods, 42(4), 1096-1104.

Stoet, G. (2017). PsyToolkit: A novel web-based method for running online questionnaires and reaction-time experiments. Teaching of Psychology, 44(1), 24-31.

Thorndike, E. L., \& Gray, B. H. (1911). Individuality.

White, R. K. (1931). The versatility of genius. The Journal of Social Psychology, 2, 460-489. https://doi.org/10.1080/00224545.1931.9918987 


\section{Appendix}

Table 1.2 - Correlations between the Personality Dimensions and the Three Polymath Dimensions and the Total Score

\begin{tabular}{ccccc}
\hline Dimension & Belief & Interests & Virtual & Score \\
\hline Openness & $.374^{*}$ & .170 & $.204^{*}$ & $.383^{*}$ \\
Conscientiousness & .081 & -.124 & .113 & .045 \\
Extraversion & .001 & -.049 & .044 & -.003 \\
Agreeableness & -.026 & .030 & .202 & .081 \\
Emotional & .072 & .122 & $.139 *$ & $160 *$ \\
Stability & & & & \\
\hline
\end{tabular}

* indicates a significant correlation

Table 1.3 - Correlations between the Generalized Self-Efficacy Scale and the Three Polymath Dimensions and the Total Score

\begin{tabular}{ccccc}
\hline Dimension & Belief & Interests & Virtual & Score \\
\hline Self Efficacy & $.199 *$ & $.181 *$ & .099 & $.252 *$ \\
\hline
\end{tabular}




\section{Questions Used In the Questionnaire:}

1. Learning different things does not bore me. [Interest]

2. I often find myself fascinated by a range of fields or topics that aren't explicitly relevant to my current chosen field [Interest]

3. I often make use of/audit different courses to learn different things [Virtual]

4. I believe that learning isn't limited to the classroom [Belief]

5. I spend a lot of time on sites like Youtube or listen to podcasts, learning about topics that aren't related to the one I have currently chosen [Virtual]

6. I believe that one needs to master only one field $(R)$ [Belief]

7. I believe that the secrets of the universe cannot be properly understood through just one field. [Belief]

8. I believe that one can become a genius and isn't determined by their genes or IQ [Belief]

9. Seeing my work as tasks to be completed instead of puzzles to be solved makes it easier for me to finish it. (R) [Interests]

10. I often install and make use of apps on my phone that help me grow or learn more [Virtual]

11. I tend to see a particular issue from different perspectives rather than the one offered. [Interests]

12. I only download books/articles/videos that are relevant to my field. (R) [Virtual] 


\section{Code Used To Construct The Survey (Psytoolkit)}

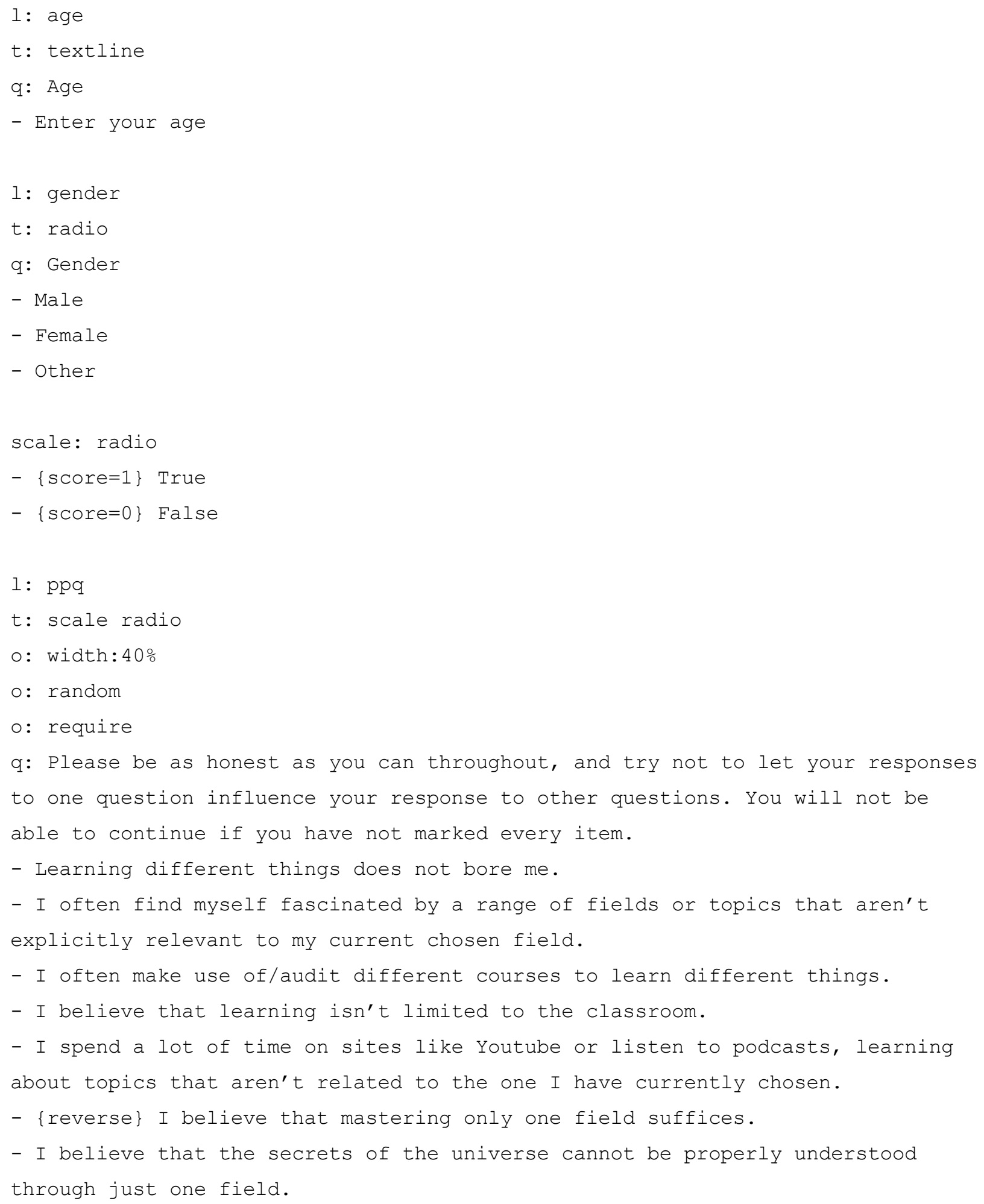


- I believe that one can become a genius and isn't determined by their genes or IQ.

- \{reverse\} Seeing my work as tasks to be completed instead of puzzles to be solved makes it easier for me to finish it.

- I often install and make use of apps on my phone that help me grow or learn more.

- I tend to see a particular issue from different perspectives rather than the one offered.

- \{reverse\} I only download books/articles/videos that are relevant to my field.

1: score1

$t:$ set

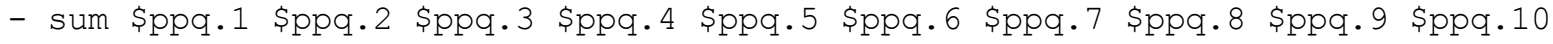
\$ppq.11 \$ppq.12

l: interest

$t:$ set

- sum \$ppq.1 \$ppq.2 \$ppq.9 \$ppq.11

1: virtual

$t$ : set

- sum \$ppq.4 \$ppq. 6 \$ppq. 7 \$ppq. 8

1: belief

$t:$ set

- sum \$ppq.3 \$ppq.5 \$ppq.10 \$ppq.12

1: feedback

$t:$ info

q: Your score is: $\{\$$ score1\}/ 12 